\title{
Selección de Aplicación para Gamificación en una Asignatura de los Grados en Ingeniería Eléctrica y Electrónica Industrial y Automática
}

\section{Carnero María Carmen}

Universidad de Castilla-La Mancha, Ciudad Real, Spain, carmen.carnero@uclm.es.

\begin{abstract}
Resumen
Debido al gran número de beneficios que reporta, el número de aplicaciones para gamificación se ha incrementado considerablemente desde 2002. Por ello, la selección de la aplicación idónea a emplear en una asignatura universitaria de grado se ha vuelto más compleja. Esta contribución pretende ayudar en esta toma de decisiones proponiendo un modelo objetivo elaborado mediante Measuring Attractiveness by a Categorical Based Evaluation Technique (MACBETH). Este modelo se ha aplicado a una asignatura de los grados en Ingeniería Eléctrica e Ingeniería Electrónica Industrial y Automática de la Escuela Técnica Superior de Ingenieros Industriales de Ciudad Real, empleándose como centro decisor uno de los profesores de la asignatura. El modelo emplea diez criterios de decisión dispuestos en una estructura jerárquica. Para cada criterio, a partir de una matriz de comparaciones por parejas construida a partir de los juicios emitidos por el centro decisor, se obtiene mediante programación lineal una función de valor. Mediante la aplicación de la metodología MACBETH, y con la ayuda del software M-MACBETH, que emplea un método simple aditivo, se obtiene una clasificación completa de las diferentes aplicaciones para gamificación evaluadas.
\end{abstract}

Palabras Claves: Gamificación, Toma de decisiones, MACBETH.

\section{Introducción}

Frente al modelo tradicional de enseñanza basado en la lección magistral y actitud pasiva de los estudiantes, las nuevas tendencias en educación defienden metodologías activas y participativas de los estudiantes (Corchuelo-Rodriguez, 2018). En este sentido, la gamificación se muestra como una herramienta que adapta las mecánicas y elementos del 
juego a la educación (Rodríguez \& Santiago, 2015). Los beneficios de aplicar gamificación a docencia son numerosos (Hurtado, 2017): incrementar la implicación y motivación de los participantes, integrar teoría y práctica, la retroalimentación es inmediata por lo que errar motiva a volver a intentarlo, hacer divertido el aprendizaje de conceptos o materias difíciles, mejorar dinámicas de grupo, promover una competitividad sana, aumentar el empleo de la creatividad, desarrollar las competencias digitales, ayudar en la resolución de problemas, promover la interacción con otros estudiantes, incrementar el interés por participar en las aulas, etc. Además, la gamificación evalúa al estudiante de una forma innovadora ya que permite valorar el alcance del conocimiento durante el proceso de aprendizaje y no solamente aportar una calificación final (Acuña, 2018).

Existe numerosas contribuciones que muestran experiencias positivas en el uso de gamificación en asignaturas universitarias, entre ellas se encuentra la desarrollada por Serna, Mauricio, San Miguel y Megías, (2016) que aplicaron gamificación a los estudiantes de Grado de Odontología de la Universidad de Valencia y, como resultado, apreciaron que al 97,30\% de los estudiantes les había ayudado a entender mejor los conceptos, el 95,95\% expusieron que era más fácil mantener la atención que si se utilizasen clases teóricas/prácticas tradicionales y, el 83,78\% apoyaron el hecho de que repasar la materia en grupo había sido más provechoso que hacerlo de forma individual. Corchuelo-Rodriguez (2018) la aplicó entre 89 estudiantes de pregrado de la Universidad de La Sabana empleando ClassDojo, Kahoot! y Educaplay; se aprecia que el 96,2\% de los estudiantes considera que es útil, así como la aceptación positiva de los estudiantes es del 89\%; además, el 88\% de los encuestados indicó que es una metodología motivante. Morís (2017) describe su experiencia en la asignatura Fundamentos Biológicos del Lenguaje del Grado en Logopedia de la Universidad de Oviedo, en la que empleó Kahoot! y, posteriormente efectúo una encuesta para conocer el grado de satisfacción de los estudiantes. Entre los resultados se puede destacar que se había adquirido, con respecto a una clase expositiva clásica: más conocimiento $(62,50 \%)$, mayor motivación $(91,67 \%)$, superior cooperación entre alumnos $(70,83 \%)$, más interés en asistir a clase $(75,00 \%)$, o una valoración global buena de las clases con tecnologías innovadoras $(83,33 \%)$. Sin embargo, hay que cuidar el diseño, planeación e implementación de la estrategia de gamificación ya que por ejemplo en la experiencia en la Universidad de Barcelona Taller de creación de JOCS, los estudiantes disminuyeron su nivel académico en la asignatura (Corchuelo-Rodriguez, 2018).

Además, la gamificación ya se encuentra presente en grandes empresas como SEAT, KPMG, Chiesi y Novartis. Así se ha contabilizado que un 55\% de las compañías más empleadoras en España ha implantado metodologías relacionadas con la gamificación (Martínez, 2016). Este empleo se ha efectuado en marketing, recursos humanos, destinado a la formación de trabajadores o altos directivos, o en la gestión de las 
relaciones con los clientes. Por tanto, para los estudiantes universitarios supone un gran avance conocer estas metodologías que también estarán presentes durante su futuras actividades laborales.

Todos estos beneficios y éxitos en su aplicación han hecho que se incremente considerablemente el número de aplicaciones destinadas a gamificación, así se pueden encontrar las siguientes: Arcademics, Brainscape, Knowre, Cerebriti, Minecraft: Education Edition, Pear Deck, Kahoot!, Edmodo Gamificación, CodeCombat, ClassDojo, Triventy Genially, Quizlet, Toovari, The World Peace Game, Play Brighter, Quizizz, Classcraft, Trivinet, etc. (Educación 3.0, 2018). Este número de aplicaciones se prevé que se incrementará en el futuro (Rodríguez \& Santiago, 2015). El gran número de aplicaciones dificulta la elección de la más idónea para una asignatura. Por tanto, en esta contribución se ha desarrollado un modelo multicriterio mediante la metodología Measuring Attractiveness by a Categorical Based Evaluation Technique (MACBETH) para la selección de la aplicación para gamificación más conveniente destinada a la asignatura Sistemas de Fabricación y Organización Industrial, destinada en concreto al bloque de Organización Industrial de los grados en Ingeniería Eléctrica e Ingeniería Electrónica Industrial y Automática impartidas en la ETS Ingenieros Industriales de Ciudad Real.

\section{Modelo para la selección de aplicación para gamificación en una asignatura de los Grados en Ingeniería Eléctrica y Electrónica Industrial y Automática}

\subsection{Metodología MACBETH}

La metodología MACBETH fue creada por Bana e Costa y Vansnick (1997) y permite evaluar las alternativas a partir únicamente de juicios cualitativos sobre la diferencia de atractivo entre dos elementos. Además, facilita una toma de decisiones objetiva para lo que requiere una serie de pasos como la definición de indicadores asociados a cada criterio, definición de niveles de escala para cada indicador que faciliten la evaluación de las alternativas, construcción de funciones de valor que garanticen una comparación de los criterios en una escala común, validación de las asignaciones a efectuar en cada alternativa y garantiza que los juicios empleados en la metodología son consistentes. Además, la técnica dispone para su aplicación del software M-MACBETH. 


\subsection{Estructuración}

Como centro decisor se ha empleado uno de los profesores de la asignatura.

Para cada criterio se ha definido un descriptor, que permitirá valorar las alternativas en cada criterio a partir del establecimiento de varios niveles de escala. Dentro de los niveles de escala se deben identificar dos niveles de referencia: neutro $(\mathrm{N})$, calificado por el decisor ni satisfactorio ni insatisfactorio, y bueno (B) que es considerado por el decisor un nivel completamente satisfactorio (Bana e Costa \& Chagas, 2004). En esta investigación se han empleado descriptores construidos, que son aquellos elaborados a partir de combinaciones de estados de varios niveles de escala.

Después de analizar la literatura sobre gamificación, se han establecido los siguientes criterios de decisión:

- Calidad de la biblioteca de preguntas y foro de experiencias (C1). Se evalúa si la aplicación dispone de una biblioteca con calidad y cantidad de cuestionarios públicos disponibles para su utilización, así como que los cuestionarios puedan compartirse, duplicarse y editarse. Se considera también la posibilidad de disponer de un foro activo en la red que intercambio de experiencias e información

- Capacidad de competición por equipos (C2). Analiza la opción de interactuar en equipos en el desarrollo de cuestionarios, permitiendo la competición en modo pizarra.

- Capacidad para aplicar Just-in-Time-Teaching (JITT) (C3). Se evalúa la posibilidad de efectuar un JITT débil (preguntas abiertas sobre estudio previo, preguntas sobre lo que se comprende o no y sobre lo que insistir o reforzar), así como el JITT fuerte (preguntas cerradas, directas a comprobar el conocimiento sobre contenidos, para evaluarlos).

- Control del ritmo de cumplimentación del cuestionario (C4). Se analiza si el ritmo de cumplimentación del cuestionario por el estudiante puede ser controlado por el profesor que dispone de las opciones de marcar un tiempo límite o que cada alumno pueda cumplimentar el cuestionario a su ritmo con un tiempo ilimitado y el número de veces que quiera (no hay que esperar las respuestas de los demás).

- Elementos de gamificación (diversión) con impacto/motivación en el alumnado (C5). Se evalúa la capacidad de la aplicación de incluir elementos de gamificación propios de juegos, como por ejemplo que cada estudiante pueda desarrollar su propio avatar o hacer uso de los que dispone la aplicación, mensajes de ánimo y apoyo (memes), posibilidad de añadir imágenes, embeber videos de Youtube o añadir música a las preguntas, además, se muestra una clasificación final de participantes divertida

- Flexibilidad en la creación de cuestionarios (C6). Se evalúan la flexibilidad de las aplicaciones para poder incluir diferentes tipos de preguntas (verdadero/falso, preguntas cortas, etc.), posibilidad de puntuar cada pregunta de forma independiente, número de respuestas con cada pregunta, opción de incluir preguntas con imágenes o videos. 
- Obtención de resultados e informes (C7). Se evalúa la capacidad de obtener informes así como de mostrar resultados de los participantes durante el juego. La opción de ocultar nombres se ha considerado ya que en algunos casos, los estudiantes prefieren que no se conozcan los resultados obtenidos por cada uno.

- Valoración de las preguntas del cuestionario (C8). Se evalúa si el profesor puede asignar una puntuación independiente a cada pregunta o bien, se valora cada pregunta en función del número de aciertos, tiempo empleado a contestar, etc.

- Versatilidad de utilización en clase mediante dispositivos informáticos y necesidad de equipos auxiliares (C9). Se analizan la versatilidad de aplicación en clase a través de diferentes dispositivos como móvil, tablet o portátil y la necesidad de equipos auxiliares para el lanzamiento de cuestionarios como por ejemplo videoproyectores.

- Limitaciones en las versiones gratuitas (C10). Se analiza el número de usuarios que pueden desarrollar una actividad simultáneamente y las funcionalidades que no se incluyen en las versiones gratuitas. Puesto que la asignatura cuenta con unos 70-80 alumnos por curso académico, al ser común a dos grados, este criterio es relevante ya que garantizaría poder efectuar los cuestionarios entre todos los estudiantes matriculados y además no tener problemas por la restricción de funcionalidades en la versión gratuita.

En la Tabla 1 se muestra, a modo de ejemplo, el descriptor asociado al criterio Limitaciones en las versiones gratuitas. La estructura jerárquica del problema se muestra en la Fig. 1.

Tabla 1. Descriptor del criterio Limitaciones en las versiones gratuitas.

\begin{tabular}{cc}
\hline Nivel de escala & Descripción \\
\hline N1 (Bueno) & No se limita el número de usuarios por sesión ni las funcionalidades de \\
la aplicación.
\end{tabular}

Font: Elaboración propia 
Para construir la matriz de juicios entre los niveles de escala de un descriptor, se solicita al decisor juicios sobre la diferencia de atractivo entre los niveles de C10 (véase la Tabla 1). Cada vez que se incluye una respuesta en la matriz, se testea la consistencia de los juicios anteriores. Se aplica el mismo proceso a cada descriptor.

El software M-MACBETH genera, mediante programación lineal, una función de valor que asigna las valoraciones 0 y 100 a los niveles de referencia del descriptor neutro y bueno respectivamente (Bana e Costa \& Chagas, 2004). En la Fig. 2 se muestra la matriz de juicios MACBETH del criterio Limitaciones en las versiones gratuitas y su función de valor.

Todas las matrices de juicios emitidas por el decisor son consistentes. En todos los criterios se han obtenido funciones de valor con escalas numéricas lineales continuas debido a que los descriptores empleados son cualitativos; dichas funciones fueron finalmente validadas.

\begin{tabular}{|l|l|}
\hline C Selección de aplicación para gamificación \\
\hline & Cobtención de resultados e informes \\
\hline & Flexibilidad en la creación de cuestionarios \\
\hline Elementos de gamificación (diversión) con impacto/motivación en el alumnado \\
\hline Calidad de la biblioteca de preguntas y foro de experiencias \\
\hline Control del ritmo de cumplimentación del cuestionario \\
\hline Valoración de las preguntas del cuestionario \\
\hline Versatilidad de utilización en clase mediante dispositivos informáticos y necesidad de equipos auxiliares \\
\hline Capacidad de competición por equipos \\
\hline
\end{tabular}

Fig. 1. Estructura jerárquica.

Fuente: elaboración propia

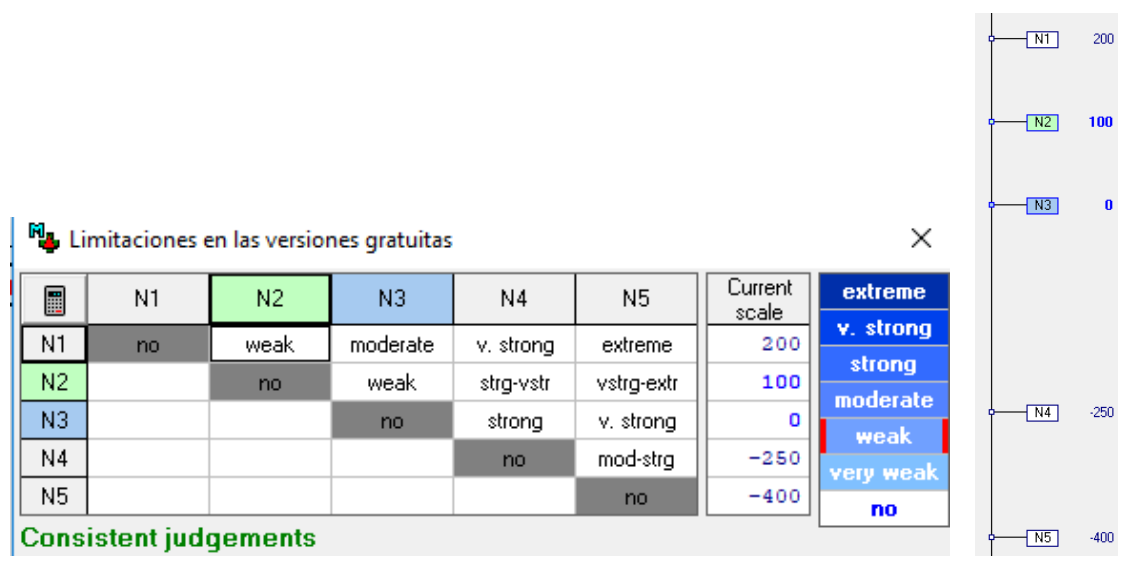

Fig. 2 Matriz de juicios MACBETH del criterio Limitaciones en las versiones gratuitas y función de valor. Fuente: elaboración propia 


\subsection{Ponderaciones}

Para obtener la ponderación de los criterios, se definió una alternativa que se encontrase en el nivel neutro en todos los criterios y subcriterios. El decisor fue preguntado, empleando las categorías semánticas MACBETH, el incremento en atractivo global proporcionado por un cambio del nivel neutro al bueno en cada criterio. A continuación el decisor comparó cuanto más preferible era el cambio desde el neutro al nivel bueno en el criterio $\mathrm{C} 5$ con respecto al mismo cambio en el criterio C10. Esta comparación se repite entre el criterio C5 y el criterio C8 y así sucesivamente. Los juicios emitidos y las ponderaciones, normalizadas en porcentajes, se muestran en la Fig. 3.

\begin{tabular}{|c|c|c|c|c|c|c|c|c|c|c|c|c|c|}
\hline 國 & [C5] & {$[\mathrm{C10}]$} & [C8] & [C6] & [C7] & [C9] & [C3] & [C1] & [C2] & [C4] & [ all low ] & $\begin{array}{l}\text { Current } \\
\text { scale }\end{array}$ & extreme \\
\hline$[\mathrm{C5}]$ & no & weak & weak-mod & weak & weak-mod & vweak-weak & weak-mod & moderate & strong & strg-vstr & positive & 16.92 & v. strong \\
\hline$[\mathrm{C} 10]$ & & no & weak & weak & weak-mod & wweak-weak & weak-mod & moderate & strong & strg-vstr & positive & 15.38 & $\begin{array}{c}\text { strong } \\
\text { moderate }\end{array}$ \\
\hline$[\mathrm{CB}]$ & & & no & vweak-weak & weak-mod & wweak-weak & weak-mod & moderate & strong & strong & positive & 13.84 & moderate \\
\hline [C6] & & & & no & no & no & weak & mod-strg & strong & strong & positive & 12.31 & dok \\
\hline [C7] & & & & no & no & no & weak & mod-strg & strong & strong & positive & 12.31 & no \\
\hline [C9] & & & & no & no & no & weak-mod & mod-strg & mod-strg & strong & positive & 12.31 & \\
\hline [C3] & & & & & & & no & weak & weak-mod & mod-strg & positive & 9.23 & \\
\hline [C1] & & & & & & & & no & wweak-weak & weak-mod & positive & 6.16 & \\
\hline [C2] & & & & & & & & & no & no & positive & 0.77 & \\
\hline [C4] & & & & & & & & & no & no & positive & 0.77 & \\
\hline [ all low] & & & & & & & & & & & no & 0.00 & \\
\hline
\end{tabular}

Fig. 3 Matriz de juicios MACBETH para los criterios

Fuente: elaboración propia

\section{Resultados}

La valoración $V(A)$ de una alternativa $A$ se obtiene aplicando la ecuación (1).

$$
V(A)=\sum_{i=1}^{n} w_{i} v_{i}(\text { impacto de } A \text { en el criterio } i)
$$

Con $\sum_{i=1}^{n} w_{i}=1 ; w_{i}>0$

$y\left\{\begin{array}{c}v_{i}(\text { nivel de impacto con nivel de escala bueno en } i)=100 \\ v_{i}(\text { nivel de impacto con nivel de escala neutro en } i)=0\end{array}\right.$

donde se están considerando $n$ criterios de decision, $w_{i}$ es la ponderación de cada criterio y $v_{i}$ (impacto de $A$ en el criterio $i$ ) es la valoración de A en el criterio $i$.

Las aplicaciones para gamificación evaluadas son: Kahoot! (K), Quizizz (Q) y Socrative (S). Las valoraciones finales obtenidas son: 


$$
\begin{array}{rl}
V(Q)=0,0616 * & 0+0,0077 * 100+0,0923 *(-233,33)+0,077 *(-150)+0,1692 \\
& * 100+0,1231 * 50+0,1231 * 60+0,1384 * 50+0,1231 * 100 \\
& +0,1538 * 50=35,46 \\
V(S)=0,0616 * & (-133,33)+0,0077 * 0+0,0923 * 100+0,077 * 100+0,1692 * 0 \\
& +0,1231 * 100+0,1231 * 100+0,1384 * 0+0,1231 * 100 \\
& +0,1538 *(-125)=19,49 \\
V(K)=0,0616 * & 100+0,0077 * 0+0,0923 *(-100)+0,077 *(-250)+0,1692 * 0 \\
& +0,1231 * 0+0,1231 * 0+0,1384 * 0+0,1231 * 0+0,1538 * 0 \\
& =-5,22
\end{array}
$$

Por tanto, la aplicación para gamificación idónea propuesta por el modelo es Quizizz.

El análisis de sensibilidad de los resultados muestra que cuando se varían ligeramente las ponderaciones de los criterios hasta valores considerados factibles por el decisor, no se modifica la clasificación de alternativas.

\section{Referencias}

Acuña, M. (2018). Las 5 mejores herramientas de gamificación para universitarios. Consultado 16 de mayo de 2019 en https://www.evirtualplus.com/herramientas-de-gamificacion-parauniversitarios/.

Bana e Costa C.A., \& Chagas, M.P. (2004). A career choice problem: an example of how to use MACBETH to build a quantitative value model based on qualitative value judgments. Eur. $J$. Oper. Res 153(2):323-331.

Bana e Costa, C. A., \& Vansnick, J. C. (1997). Applications of the MACBETH approach in the framework of an additive aggregation model. Journal of Multi-Criteria Decision Analysis, 6, 107114.

Bana e Costa, C. A., De Corte, J. M., \& Vansnick, J. C. (2011). MACBETH (Measuring Attractiveness by a Categorical Based Evaluation Technique). In J. J. Cochran, L. A. Cox, Jr., P. Keskinocak, J. P. Kharoufeh, \& J. C. Smith (Eds.), Encyclopedia of Operations Research and Management Science. New York: John Wiley \& Sons.

Corchuelo-Rodriguez C. A. (2018). Gamificación en Educación Superior: Experiencia Innovadora para Motivar Estudiantes y Dinamizar Contenidos en el Aula, Edutec, 63, 29-41.

Educación 3.0. (2018). 20 herramientas de gamificación para clase que engancharán a tus alumnos.

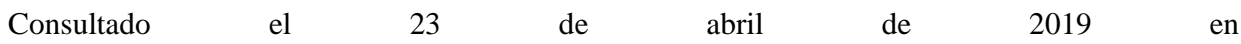
https://www.educaciontrespuntocero.com/recursos/herramientas-gamificacioneducacion/33094.html.

Hurtado, S. (2017). Gamificación: que es y cuáles son sus ventajas. Consultado el 23 de marzo de 2018 en http://es.eadbox.com/gamificacion-que-es-y-cuales-son-sus-ventajas/.

Martínez, E. (2016). La utilización de la gamificación para la formación aumenta un 18\% en España, RRHH Digital. Consultado el 16 de mayo de 2019 en 
http://www.rrhhdigital.com/secciones/tecnologia-e-innovacion/120597/La-utilizacion-de-lagamificacion-para-la-formacion-aumenta-un-18-en-Espana.

Morís, G. (2017). Gamificación a través de Kahoot como innovación docente en el Grado de Logopedia, INNODOCT 2017, Valencia, 25-27 october 2017, 705-714.

Rodríguez, F., \& Santiago, R. (2015). Gamificación: Cómo motivar a tu alumnado y mejorar el clima en el aula. Innovación educativa. Barcelona: Editorial Océano.

Serna, E., Mauricio, M. D., San Miguel, T., \& Megías, J. (2016). Experiencia de gamificación en Docencia Universitaria: aprendizaje activo y entretenido . Congreso In-Red 2016 UPV, 7 y 8 de julio de 2016, Doi: http://dx.doi.org/10.4995/INRED2016.2016.4292. 